\title{
Model Study of Three-Body Forces in the Three-Body Bound State
}

\author{
H. Liu, Ch. Elster \\ Institute of Nuclear and Particle Physics, and Department of Physics, \\ Ohio University, Athens, $\mathrm{OH} 45701$ \\ W. Glöckle \\ Institute for Theoretical Physics II, Ruhr-University Bochum, D-44780 Bochum, Germany.
} (July 22, 2013)

\begin{abstract}
The Faddeev equation for the three-body bound state with two- and threebody forces is solved directly as three-dimensional integral equation. The numerical feasibility and stability of the algorithm, which does not employ partial wave decomposition is demonstrated. The three-body binding energy and the full wave function are calculated with Malfliet-Tjon-type two-body potentials and scalar two-meson exchange three-body forces. For two and three body forces of ranges and strengths typical of nuclear forces the single particle momentum distribution and the two-body correlation function are similar to the ones found for realistic nuclear forces.
\end{abstract}

PACS number(s): $21.45+\mathrm{v}$

Typeset using REVTEX 


\section{INTRODUCTION}

Three-body Faddeev equations for the bound state using local forces directly without finite rank expansion have been solved since the pioneering work by Malfliet and Tjon [1] and Osborn [2]. After this, a huge body of work by different groups followed, and calculations using the Faddeev equations were either performed in momentum space, see e.g. [3-6], in configuration space [7-9], or in a hybrid fashion using both spaces [10]. However, the technique was always based on an angular momentum decomposition, which including spin and isospin degrees of freedom lead to quite a large set of states. For instance allowing NN forces to act in all states of total angular momenta $j$ up to $j=6$, which is necessary to control the ${ }^{3} \mathrm{H}$ binding energy within $2 \mathrm{keV}$ require 102 angular momentum and isospin combinations (in the literature often called channels). In view of this very large number of interfering terms and having in mind that computer resources are still increasing steadily, it appears natural to give up such an expansion and work directly with momentum vectors or position vectors in configuration space as it is common in Greens Function Monte Carlo methods [11]. We started an approach based on momentum vectors as variables for the three-boson bound state [12], which then could be extended to the three-boson continuum in a straightforward fashion [13]. In configuration space Faddeev equations have been solved applying vector variables for pure Coulomb bound-state problems, namely the $e^{-} e^{-} e^{+}$and the $p p \mu^{-}$systems [14]. Including spin and isospin degrees of freedom together with realistic nucleon-nucleon (NN) forces, a scheme based on momentum vectors has been applied to NN scattering leading to integral equations in two variables [15], which can easily be solved on present day computers.

Angular momentum decompositions even for three bosons require quite a tedious algebra and care in a computational implementation [16] due to often nontrivial cancellations between partial wave terms. This is especially true if one includes three-body forces $[17,18]$. In contrast, the formulation in terms of momentum vectors of the one Faddeev equation for identical particles is very straightforward and transparent. In this paper we extend the approach from Ref. [12] and include three-body forces for an investigation of the three-body bound state. This study is meant only to demonstrate the ease in solving the Faddeev equation including three-body forces, and working directly with momentum vector variables. Since we do not yet include spin and isospin degrees of freedom we do not address physical questions directly related to the physical three nucleon system, but the numerical examples given can shed some light on the wave function properties of three nucleons.

The paper is organized as follows. In Section II the Faddeev equation including two- and three-body forces is formulated in terms of momentum vectors, and its solution, especially the intermediate integrations are layed out in detail. Section III displays our choice of forces and provides the necessary numerical insight for achieving an accurate solution. Wave function properties are displayed in Section IV. Finally we summarize in Section V and provide an outlook.

\section{THREE-BODY BOUND STATE EQUATION WITH THREE-BODY FORCE}

The bound state of three identical particles which interact via pairwise forces $V^{i}=V_{j k}$ $\left(i, j, k=1,2,3\right.$ and cyclic permutations thereof) and a genuine three-body force $V_{123}$ is given 
by the Schrödinger equation which reads in integral form

$$
|\Psi\rangle=G_{0}\left(\sum_{i=1}^{3} V^{i}+V_{123}\right)|\Psi\rangle .
$$

Here the free propagator is given by $G_{0}=\left(E-H_{0}\right)^{-1}$, where $H_{0}$ stands for the free Hamiltonian and $E$ for the binding energy of the three-body system. Any three-body force $V_{123}$ can be decomposed into three different pieces as

$$
V_{123}=\sum_{i=1}^{3} V_{4}^{(i)},
$$

such that $V_{4}^{(i)}$ is symmetric under the exchange of particles $j$ and $k(j \neq i \neq k)$. The decomposition suggested in Eq. (2.2) is natural for e.g. realistic $\pi \pi 3 \mathrm{~N}$ forces, which are considered at present in all available $3 \mathrm{~N}$ forces. Introducing Faddeev components $|\Psi\rangle=$ $\sum_{i=1}^{3}\left|\psi_{i}\right\rangle$ with

$$
\left|\psi_{i}\right\rangle=G_{0}\left(V_{i}+V_{4}^{(i)}\right)|\Psi\rangle
$$

leads to three coupled integral equations

$$
\begin{aligned}
\left|\psi_{i}\right\rangle & =G_{0} t_{i} \sum_{j \neq i}\left|\psi_{j}\right\rangle+\left(1+G_{0} t_{i}\right) G_{0} V_{4}^{(i)} \sum_{j}\left|\psi_{j}\right\rangle \\
& =G_{0}\left\{t_{i} \sum_{j \neq i}\left|\psi_{j}\right\rangle+\left(1+t_{i} G_{0}\right) V_{4}^{(i)} \sum_{j}\left|\psi_{j}\right\rangle\right\} .
\end{aligned}
$$

The operator $t_{i}$ describes the two-body $t$-matrix in the subsystem $j k$. If we consider identical particles (here bosons, since we are omitting spin), the three-nucleon wave function $|\Psi\rangle$ has to be totally symmetric. As a consequence, the Faddeev components $\left|\psi_{i}\right\rangle$ are identical in their functional form, only the particles are permuted. Thus it is sufficient to consider only one component, e.g.

$$
\left|\psi_{1}\right\rangle=G_{0} t_{1} P\left|\psi_{1}\right\rangle+\left(1+G_{0} t\right) V_{4}^{(1)}(1+P)\left|\psi_{1}\right\rangle
$$

In the following the index (1) will be dropped. The permutation operator $P$ is given as $P=P_{12} P_{23}+P_{13} P_{23}$ and the total wave function reads

$$
|\Psi\rangle=(1+P)|\psi\rangle
$$

In order to solve Eq. (2.5) standard Jacobi momenta are used,

$$
\begin{aligned}
\mathbf{p}_{i} & =\frac{1}{2}\left(\mathbf{k}_{j}-\mathbf{k}_{k}\right) \\
\mathbf{q}_{i} & =\frac{2}{3}\left(\mathbf{k}_{i}-\frac{1}{2}\left(\mathbf{k}_{j}+\mathbf{k}_{k}\right)\right),
\end{aligned}
$$

where $i j k=123$ and cyclic permutations thereof. For later clarification we label the coordinates with $i j k=123$ as system of 'type (1)', the ones with $i j k=231$ as 'type (2)' and the 
ones with $i j k=312$ as 'type (3)'. With the Jacobi momenta from Eq. (2.7) and omitting the arbitrarily chosen index 1, Eq. (2.5) reads

$$
\langle\mathbf{p q} \mid \psi\rangle=\frac{1}{E-\frac{p^{2}}{m}-\frac{3 q^{2}}{4 m}}\left\langle\mathbf{p q}\left|t P+V_{4}(1+P)+t G_{0} V_{4}(1+P)\right| \psi\right\rangle .
$$

Introducing the symmetrized two-nucleon $t$-matrix

$$
t_{s}(\mathbf{p}, \mathbf{q} ; E)=t(\mathbf{p}, \mathbf{q} ; E)+t(-\mathbf{p}, \mathbf{q} ; E)
$$

and explicitly working out the permutation operator $P$ in the first term of Eq. (2.8) leads to

$$
\begin{aligned}
\langle\mathbf{p q} \mid \psi\rangle & =\frac{1}{E-\frac{p^{2}}{m}-\frac{3 q^{2}}{4 m}}\left[\int d^{3} q^{\prime} t_{s}\left(\mathbf{p}, \frac{1}{2} \mathbf{q}+\mathbf{q}^{\prime} ; E-\frac{3}{4 m} q^{2}\right)\left\langle\mathbf{q}+\frac{1}{2} \mathbf{q}^{\prime}, \mathbf{q}^{\prime} \mid \psi\right\rangle\right. \\
& \left.+\left\langle\mathbf{p q}\left|V_{4}(1+P)\right| \psi\right\rangle+\frac{1}{2} \int d^{3} \tilde{p} \frac{t_{s}\left(\mathbf{p}, \tilde{\mathbf{p}} ; E-\frac{3}{4 m} q^{2}\right)}{E-\frac{\tilde{p}^{2}}{m}-\frac{3}{4 m} q^{2}}\left\langle\tilde{\mathbf{p}} \mathbf{q}\left|V_{4}(1+P)\right| \psi\right\rangle\right]
\end{aligned}
$$

A three-body force (3BF) with two scalar meson exchanges and a constant meson-nucleon amplitude can be written in the form of Eq. (2.2) with

$$
V_{4} \equiv V_{4}^{(1)} \propto \frac{F\left(Q^{2}\right)}{Q^{2}+m_{s}^{2}} \frac{F\left(Q^{\prime 2}\right)}{Q^{\prime 2}+m_{s}^{2}}
$$

and a cutoff function

$$
F\left(Q^{2}\right)=\left(\frac{\Lambda^{2}-m_{s}^{2}}{\Lambda^{2}+Q^{2}}\right)^{2}
$$

The momentum transfer $\mathbf{Q}\left(\mathbf{Q}^{\prime}\right)$ is given by

$$
\begin{gathered}
\mathbf{Q}=\mathbf{k}_{3}-\mathbf{k}_{3}^{\prime}=\mathbf{p}-\mathbf{p}^{\prime}-\frac{1}{2}\left(\mathbf{q}-\mathbf{q}^{\prime}\right) \\
\mathbf{Q}^{\prime}=\mathbf{k}_{2}^{\prime}-\mathbf{k}_{2}=\mathbf{p}-\mathbf{p}^{\prime}+\frac{1}{2}\left(\mathbf{q}-\mathbf{q}^{\prime}\right),
\end{gathered}
$$

as indicated in Fig. 1.

For the evaluation of Eq. (2.10) matrix elements of the form $\left\langle\mathbf{p q}\left|V_{4}(1+P)\right| \psi\right\rangle$ need to be calculated. From Fig. 1 we see that $V_{4}$ can be considered as a sequence of meson exchanges in the subsystem (12), called for convenience subsystem 3, and subsystem (31), called 2. Inserting a complete set of states of the type 3 between $V_{4}$ and $(1+P)|\psi\rangle$ and another complete set of states of type 2 between the two meson exchanges (see Eq. (2.11)), leads after a straightforward evaluation to

$$
\begin{aligned}
\left\langle\mathbf{p q}\left|V_{4}(1+P)\right| \psi\right\rangle & =\int d^{3} q^{\prime} \frac{F\left(\left(-\mathbf{p}-\frac{1}{2} \mathbf{q}-\mathbf{q}^{\prime}\right)^{2}\right)}{\left(-\mathbf{p}-\frac{1}{2} \mathbf{q}-\mathbf{q}^{\prime}\right)^{2}+m_{s}^{2}} \\
& \times \int d^{3} p^{\prime} \frac{F\left(\left(-\mathbf{p}+\frac{1}{2} \mathbf{q}-\frac{1}{2} \mathbf{q}^{\prime}-\mathbf{p}^{\prime}\right)^{2}\right)}{\left(-\mathbf{p}+\frac{1}{2} \mathbf{q}-\frac{1}{2} \mathbf{q}^{\prime}-\mathbf{p}^{\prime}\right)^{2}+m_{s}^{2}}\left\langle\mathbf{p}^{\prime} \mathbf{q}^{\prime}|(1+P)| \psi\right\rangle
\end{aligned}
$$


The propagators in Eq. (2.14) contain linear combinations of three or four momentum vectors and thus the two integrations would involve magnitudes of vectors and angles between them. Realizing that both meson-exchange propagators in the 3BF term only depend on the momentum transfer in a two-body subsystem, one can rewrite Eq. (2.14) as

$$
\begin{aligned}
\left\langle\mathbf{p q}\left|V_{4}(1+P)\right| \psi\right\rangle & =\int d^{3} p^{\prime} d^{3} q^{\prime}\left\langle\mathbf{p q} \mid \mathbf{p}^{\prime} \mathbf{q}^{\prime}\right\rangle_{2} \\
& \times \int d^{3} p^{\prime \prime} \frac{F\left(\left(\mathbf{p}^{\prime}-\mathbf{p}^{\prime \prime}\right)^{2}\right)}{\left(\mathbf{p}^{\prime}-\mathbf{p}^{\prime \prime}\right)^{2}+m_{s}^{2}} \\
& \times \int d^{3} p^{\prime \prime \prime} d^{3} q^{\prime \prime \prime}{ }_{2}\left\langle\mathbf{p}^{\prime \prime} \mathbf{q}^{\prime} \mid \mathbf{p}^{\prime \prime \prime} \mathbf{q}^{\prime \prime \prime}\right\rangle_{3} \\
& \times \int d^{3} p^{\prime \prime \prime \prime \prime} \frac{F\left(\left(\mathbf{p}^{\prime \prime \prime}-\mathbf{p}^{\prime \prime \prime \prime}\right)^{2}\right)}{\left(\mathbf{p}^{\prime \prime \prime}-\mathbf{p}^{\prime \prime \prime \prime}\right)^{2}+m_{s}^{2}}{ }_{3}\left\langle\mathbf{p}^{\prime \prime \prime \prime} \mathbf{q}^{\prime \prime \prime \prime} \mid \Psi\right\rangle .
\end{aligned}
$$

Here the subscripts 1, 2, 3 of the bra and ket vectors stand for the different types of coordinate systems described by Eq. (2.7). Though the integrations of Eq. (2.15) look more complicated, the meson-propagators show a very simple form. In fact, the integrations over $\mathbf{p}^{\prime \prime}$ and $\mathbf{p}^{\prime \prime \prime \prime}$ have an identical functional form. Defining

$$
F_{3}\left(\mathbf{p}^{\prime \prime \prime}, \mathbf{q}^{\prime \prime \prime}\right)=\int d^{3} p^{\prime \prime \prime \prime \prime} \frac{F\left(\left(\mathbf{p}^{\prime \prime \prime}-\mathbf{p}^{\prime \prime \prime \prime}\right)^{2}\right)}{\left(\mathbf{p}^{\prime \prime \prime}-\mathbf{p}^{\prime \prime \prime \prime}\right)^{2}+m_{s}^{2}}{ }_{3}\left\langle\mathbf{p}^{\prime \prime \prime \prime} \mathbf{q}^{\prime \prime \prime} \mid \Psi\right\rangle,
$$

the integration of the meson exchange between particles 2 and 1 in Eq. (2.16) is carried out completely in the coordinate system of type 3 . Once $F_{3}\left(\mathbf{p}^{\prime \prime \prime}, \mathbf{q}^{\prime \prime \prime}\right)$ is obtained, it needs to be expressed in terms of momenta in a coordinate system of type 2 in order to carry out the integration over the remaining meson exchange. This transformation, labeled $F_{32}\left(\mathbf{p}^{\prime \prime}, \mathbf{q}^{\prime}\right)$ is explicitly given as

$$
\begin{aligned}
F_{32}\left(\mathbf{p}^{\prime \prime}, \mathbf{q}^{\prime}\right) & =\int d^{3} p^{\prime \prime \prime} d^{3} q^{\prime \prime \prime}{ }_{2}\left\langle\mathbf{p}^{\prime \prime} \mathbf{q}^{\prime} \mid \mathbf{p}^{\prime \prime \prime} \mathbf{q}^{\prime \prime \prime}\right\rangle_{3} F_{3}\left(\mathbf{p}^{\prime \prime \prime}, \mathbf{q}^{\prime \prime \prime}\right) \\
& =F_{3}\left(\left|-\frac{1}{2} \mathbf{p}^{\prime \prime}-\frac{3}{4} \mathbf{q}^{\prime}\right|,\left|\mathbf{p}^{\prime \prime}-\frac{1}{2} \mathbf{q}^{\prime}\right|, \frac{\left(-\frac{1}{2} \mathbf{p}^{\prime \prime}-\frac{3}{4} \mathbf{q}^{\prime}\right) \cdot\left(\mathbf{p}^{\prime \prime}-\frac{1}{2} \mathbf{q}^{\prime}\right)}{\left|-\frac{1}{2} \mathbf{p}^{\prime \prime}-\frac{3}{4} \mathbf{q}^{\prime}\right|\left|\mathbf{p}^{\prime \prime}-\frac{1}{2} \mathbf{q}^{\prime}\right|}\right)
\end{aligned}
$$

Here we used that $F_{3}\left(\mathbf{p}^{\prime \prime \prime}, \mathbf{q}^{\prime \prime \prime}\right)$ is a scalar function due to the total wave function $\Psi(\mathbf{p}, \mathbf{q})$ being a scalar in the ground state. The integration over the second meson exchange between particle 1 and 3 in the coordinate system of type 2 is now given by

$$
F_{2}\left(\mathbf{p}^{\prime}, \mathbf{q}^{\prime}\right)=\int d^{3} p^{\prime \prime} \frac{F\left(\left(\mathbf{p}^{\prime}-\mathbf{p}^{\prime \prime}\right)^{2}\right)}{\left(\mathbf{p}^{\prime}-\mathbf{p}^{\prime \prime}\right)^{2}+m_{s}^{2}} F_{32}\left(\mathbf{p}^{\prime \prime}, \mathbf{q}^{\prime}\right) .
$$

The matrix element $\left\langle\mathbf{p q}\left|V_{4}(1+P)\right| \psi\right\rangle$ is finally obtained by integrating $F_{2}\left(\mathbf{p}^{\prime}, \mathbf{q}^{\prime}\right)$ over $\mathbf{p}^{\prime}$ and $\mathbf{q}^{\prime}$, i.e. carrying out the final coordinate transformation from the system of type 2 back to the one of type 1 ,

$$
\begin{aligned}
\left\langle\mathbf{p q}\left|V_{4}(1+P)\right| \psi\right\rangle & =\int d^{3} p^{\prime} d^{3} q^{\prime}\left\langle\mathbf{p q} \mid \mathbf{p}^{\prime} \mathbf{q}^{\prime}\right\rangle_{2} F_{2}\left(\mathbf{p}^{\prime}, \mathbf{q}^{\prime}\right) \\
& =F_{2}\left(\left|-\frac{1}{2} \mathbf{p}+\frac{3}{4} \mathbf{q}\right|,\left|-\mathbf{p}-\frac{1}{2} \mathbf{q}\right|, \frac{\left(-\frac{1}{2} \mathbf{p}+\frac{3}{4} \mathbf{q}\right) \cdot\left(-\mathbf{p}-\frac{1}{2} \mathbf{q}\right)}{\left|-\frac{1}{2} \mathbf{p}+\frac{3}{4} \mathbf{q}\right|\left|-\mathbf{p}-\frac{1}{2} \mathbf{q}\right|}\right)
\end{aligned}
$$


Thus, the integration of Eq. (2.15), explicitly given in separate steps from Eq. (2.16) to Eq. (2.19) contains only integrations over one vector variable at a time. It should be pointed out that Eqs. (2.17) and Eq. (2.19) are only three dimensional interpolations. Clearly, the in Eq. (2.15) suggested method is the preferred one for practical calculations.

The Faddeev amplitude $\psi(\mathbf{p}, \mathbf{q})$ is given as function of vector Jacobi momenta and obtained as solution of a three dimensional integral equation, Eq. (2.10). For the ground state $\psi(\mathbf{p}, \mathbf{q})$ is also a scalar and thus only depends on the magnitudes of $\mathbf{p}$ and $\mathbf{q}$ and the angle between the two vectors. In order to solve the Eq. (2.10) directly without introducing partial wave projection, we have to define a coordinate system. We choose the vector $\mathbf{q}$ parallel to the $z$-axis and express the remaining vectors with respect to $\mathbf{q}$. For the first term in Eq. (2.10) the relevant vectors are $\mathbf{p}$ and $\mathbf{q}^{\prime}$. Thus one has aside from the magnitudes the following angle relations

$$
\begin{aligned}
x & =\hat{\mathbf{p}} \cdot \hat{\mathbf{q}}=\cos \theta \\
x^{\prime} & =\hat{\mathbf{q}}^{\prime} \cdot \hat{\mathbf{q}}=\cos \theta^{\prime} \\
y & =\hat{\mathbf{p}} \cdot \hat{\mathbf{q}}^{\prime}=\cos \gamma
\end{aligned}
$$

where

$$
\cos \gamma=\cos \theta \cos \theta^{\prime}+\sin \theta \sin \theta^{\prime} \cos \left(\phi-\phi^{\prime}\right)=x x^{\prime}+\sqrt{1-x^{2}} \sqrt{1-x^{\prime 2}} \cos \phi^{\prime}
$$

Since the $\phi^{\prime}$ integration is over the full $2 \pi$ interval we have a freedom of choice for the azimuthal angle angle and set $\phi=0$. With these choice of variables Eq. (2.10) with only the first term, i.e. two-body forces alone, was solved successfully in Ref [12]. For the evaluation of the second term in Eq. (2.10),

$$
\left\langle\mathbf{p q}\left|V_{4}(1+P)\right| \psi\right\rangle=\left\langle\mathbf{p q}\left|V_{4}\right| \Psi\right\rangle
$$

we start with calculating first $F_{3}\left(\mathbf{p}^{\prime \prime \prime}, \mathbf{q}^{\prime \prime \prime}\right)$, Eq. (2.16), and realize, that for this integration we can choose $\mathbf{q}^{\prime \prime \prime}$ parallel to the $z$-axis with corresponding simplifications for one of the azimuthal angles. This leads to the explicit expression

$$
\begin{aligned}
& F_{3}\left(p^{\prime \prime \prime}, q^{\prime \prime \prime}, x^{\prime \prime \prime}\right)= \\
& \int_{0}^{\infty} d p^{\prime \prime \prime \prime} p^{\prime \prime \prime \prime 2} \int_{-1}^{+1} d x^{\prime \prime \prime \prime} \int_{0}^{2 \pi} d \phi^{\prime \prime \prime \prime} \frac{\left(\frac{\Lambda^{2}-m_{\alpha}^{2}}{\Lambda^{2}+\left(p^{\prime \prime \prime}+p^{\prime \prime \prime \prime}-2 p^{\prime \prime \prime} p^{\prime \prime \prime \prime} y^{\prime \prime \prime \prime}\right)^{2}}\right)^{2}}{\left(p^{\prime \prime \prime}+p^{\prime \prime \prime \prime} 2-2 p^{\prime \prime \prime} p^{\prime \prime \prime \prime} y^{\prime \prime \prime \prime}\right)^{2}+m_{\alpha}^{2}} \Psi\left(p^{\prime \prime \prime \prime}, q^{\prime \prime \prime}, x^{\prime \prime \prime \prime \prime}\right) .
\end{aligned}
$$

The evaluation of $F_{32}\left(\mathbf{p}^{\prime \prime}, \mathbf{q}^{\prime}\right)$, Eq. (2.17) is not an integration but rather a three dimensional interpolation and explicitly given by

$$
\begin{aligned}
& F_{32}\left(p^{\prime \prime}, q^{\prime}, x^{\prime \prime}\right)= \\
& F_{3}\left(\frac{1}{2} \sqrt{\frac{9}{4} q^{\prime 2}+p^{\prime \prime 2}+3 p^{\prime \prime} q^{\prime} x^{\prime \prime}}, \sqrt{\frac{1}{4} q^{2}+p^{\prime \prime 2}-p^{\prime \prime} q^{\prime} x^{\prime \prime}}, \frac{\frac{3}{8} q^{\prime 2}-\frac{1}{2} p^{\prime \prime 2}-\frac{1}{2} p^{\prime \prime} q^{\prime} x^{\prime \prime}}{\left|-\frac{3}{4} \mathbf{q}^{\prime}-\frac{1}{2} \mathbf{p}^{\prime \prime}\right|\left|+\mathbf{p}^{\prime \prime}-\frac{1}{2} \mathbf{q}^{\prime}\right|}\right) .
\end{aligned}
$$

with 


$$
\begin{aligned}
\left|-\frac{3}{4} \mathbf{q}^{\prime}-\frac{1}{2} \mathbf{p}^{\prime \prime}\right| & =\frac{1}{2} \sqrt{\frac{9}{4} q^{2}+p^{\prime 2}+3 p^{\prime \prime} q^{\prime} x^{\prime \prime}} \\
\left|+\mathbf{p}^{\prime \prime}-\frac{1}{2} \mathbf{q}^{\prime}\right| & =\sqrt{\frac{1}{4} q^{\prime 2}+p^{\prime \prime 2}-p^{\prime \prime} q^{\prime} x^{\prime \prime}} .
\end{aligned}
$$

For the interpolation we apply the cubic splines introduced in Ref [18]. The integration over the second meson exchange, i.e. the calculation of $F_{2}\left(\mathbf{p}^{\prime}, \mathbf{q}^{\prime}\right)$ of Eq. (2.18) is functionally the same as Eq. (2.23), since we can choose the variable $\mathbf{q}^{\prime}$ parallel to the $z$-axis. Thus we have the same expression as Eq. (2.23) with $p^{\prime}, q^{\prime}, x^{\prime}, p^{\prime \prime}, x^{\prime \prime}$ and $\phi^{\prime \prime}$ instead of $p^{\prime \prime \prime}, q^{\prime \prime \prime}, x^{\prime \prime \prime}$, $p^{\prime \prime \prime \prime}, x^{\prime \prime \prime \prime}$ and $\phi^{\prime \prime \prime \prime}$. Finally, the matrix element $\left\langle\mathbf{p q}\left|V_{4}\right| \Psi\right\rangle$ is explicitly obtained by a second interpolation as

$$
\begin{aligned}
\left\langle\mathbf{p q}\left|V_{4}\right| \Psi\right\rangle & \equiv V_{4} \Psi(p, q, x) \\
& =F_{2}\left(\frac{1}{2} \sqrt{\frac{9}{4} q^{2}+p^{2}-3 p q x}, \sqrt{\frac{1}{4} q^{2}+p^{2}+p q x}, \frac{-\frac{3}{8} q^{2}+\frac{1}{2} p^{2}-\frac{1}{2} p q x}{\left|+\frac{3}{4} \mathbf{q}-\frac{1}{2} \mathbf{p}\right|\left|-\mathbf{p}-\frac{1}{2} \mathbf{q}\right|}\right)
\end{aligned}
$$

The last term of Eq. (2.10) requires an additional integration of the matrix element $\left\langle\mathbf{p q}\left|V_{4}(1+P)\right| \psi\right\rangle$ and the half shell two body $t$-matrix. Again, with choosing q parallel to the $z$-axis we only have three vectors to consider, $\tilde{\mathbf{p}}, \mathbf{p}$ and $\mathbf{q}$, thus the integration is of a similar type as the one of the first term in Eq. (2.10),

$$
\begin{aligned}
& \frac{1}{2} \int d^{3} \tilde{p} \frac{t_{s}\left(\mathbf{p}, \tilde{\mathbf{p}} ; E-\frac{3}{4 m} q^{2}\right)}{E-\frac{\tilde{p}^{2}}{m}-\frac{3}{4 m} q^{2}}\left\langle\tilde{\mathbf{p}} \mathbf{q}\left|V_{4}(1+P)\right| \psi\right\rangle \\
= & \frac{1}{2} \int_{0}^{\infty} d \tilde{p} \tilde{p}^{2} \int_{1}^{+1} d \tilde{x} \int_{0}^{2 \pi} d \tilde{\phi} \frac{t_{s}\left(p, \tilde{p}, \tilde{y}_{p}, ; E-\frac{3}{4 m} q^{2}\right)}{E-\frac{\tilde{p}^{2}}{m}-\frac{3}{4 m} q^{2}} V_{4} \Psi(\tilde{p}, q, \tilde{x})
\end{aligned}
$$

with

$$
\begin{aligned}
\tilde{x} & =\hat{\tilde{\mathbf{p}}} \cdot \hat{\mathbf{q}} \\
\tilde{y}_{p} & =\hat{\tilde{\mathbf{p}}} \cdot \hat{\mathbf{p}}=x \tilde{x}+\sqrt{1-x^{2}} \sqrt{1-\tilde{x}^{2}} \cos \tilde{\phi} .
\end{aligned}
$$

We obtain the energy eigenvalue $E$ and Faddeev component $\psi(p, q, x)$ of the three-body system by solving Eq. (2.10).

Finally, we want to give the explicit expression for the full wave function from Eq. (2.6), which is

$$
\begin{aligned}
\Psi(p, q, x) & =\psi(p, q, x) \\
& +\psi\left(\frac{1}{2} \sqrt{\frac{9}{4} q^{2}+p^{2}+3 p q x}, \sqrt{\frac{1}{4} q^{2}+p^{2}-p q x}, \frac{\frac{3}{8} q^{2}-\frac{1}{2} p^{2}-\frac{1}{2} p q x}{\left|-\frac{3}{4} \mathbf{q}-\frac{1}{2} \mathbf{p}\right|\left|-\frac{1}{2} \mathbf{q}+\mathbf{p}\right|}\right) \\
& +\psi\left(\frac{1}{2} \sqrt{\frac{9}{4} q^{2}+p^{2}-3 p q x}, \sqrt{\frac{1}{4} q^{2}+p^{2}+p q x}, \frac{-\frac{3}{8} q^{2}+\frac{1}{2} p^{2}-\frac{1}{2} p q x}{\left|+\frac{3}{4} \mathbf{q}-\frac{1}{2} \mathbf{p}\right|\left|-\frac{1}{2} \mathbf{q}-\mathbf{p}\right|}\right) .
\end{aligned}
$$


The wave function is normalized according to

$$
\int d^{3} p d^{3} q \Psi^{2}(\mathbf{p}, \mathbf{q})=1
$$

\section{CALCULATION OF THE THREE-BODY BOUND STATE}

Our model calculations are based on Yukawa interactions. As two-body force (2BF) we employ a Malfliet-Tjon [1] type potential

$$
\begin{aligned}
V\left(\mathbf{q}, \mathbf{q}^{\prime}\right)= & -\frac{g_{A}^{2}}{(2 \pi)^{3}} \frac{1}{\left(\mathbf{q}-\mathbf{q}^{\prime}\right)^{2}+m_{A}^{2}}\left(\frac{\Lambda_{A}^{2}-m_{A}^{2}}{\left(\mathbf{q}-\mathbf{q}^{\prime}\right)^{2}+\Lambda_{A}^{2}}\right)^{2} \\
& +\frac{g_{R}^{2}}{(2 \pi)^{3}} \frac{1}{\left(\mathbf{q}-\mathbf{q}^{\prime}\right)^{2}+m_{R}^{2}}\left(\frac{\Lambda_{R}^{2}-m_{R}^{2}}{\left(\mathbf{q}-\mathbf{q}^{\prime}\right)^{2}+\Lambda_{R}^{2}}\right)^{2},
\end{aligned}
$$

which is modified by a cutoff function of dipole type. With this choice, the $2 \mathrm{BF}$ and the 3BF have similar functional forms for the scalar meson exchanges. The force in Eq.(3.1) is a superposition of a short-ranged repulsive and long-ranged attractive Yukawa interactions. The coupling constants and exchanged meson masses are characterized by subscripts $\mathrm{R}$ and A respectively. The exchanged masses $m_{A}$ and $m_{R}$ are those from the original MalflietTjon model, the coupling constants are chosen so that the two-body force gives a binding of the three-body system which is slightly smaller than the experimental value of the triton binding, which is $8.48 \mathrm{MeV}$. The cutoff masses $\Lambda$ have values typical for one-boson-exchange models. The parameters for the 2BF, named MT2-II, are given in Table I. They lead to a two-body binding energy of $0.284 \mathrm{MeV}$, and the S-wave phase-shift roughly follows the shape of the experimental one for the state ${ }^{3} S_{1}$, though being less attractive. Since we neglect spin degrees of freedom, any closer adjustment to real phase shifts would be meaningless anyhow.

With this interaction we first solve the Lippmann-Schwinger equation for the fully-offshell two-body $t$-matrix directly as function of vector variables as described in Ref. [21]. This $t$-matrix is then symmetrized to obtain $t_{s}\left(p^{\prime}, p, x, E-\frac{3}{4 m} q^{2}\right)$. The eigenvalue equation, Eq. (2.10), for the three-body bound state is solved iteratively by a Lanczo's type algorithm described in detail in Ref. [4]. Using the 2BF alone, the binding energy of the three-body system is calculated as $E=7.6986 \mathrm{MeV}$.

The simplest $3 \mathrm{BF}$ we want to apply in our study has the functional form

$$
V_{4}=\frac{1}{(2 \pi)^{6}} \frac{a_{\alpha}}{m_{\alpha}} g_{\alpha}^{2} \frac{F_{\alpha}\left(Q^{2}\right)}{Q^{2}+m_{\alpha}^{2}} \frac{F_{\alpha}\left(Q^{\prime 2}\right)}{Q^{\prime 2}+m_{\alpha}^{2}},
$$

where

$$
F_{\alpha}\left(Q^{2}\right)=\left(\frac{\Lambda_{\alpha}^{2}-m_{\alpha}^{2}}{\Lambda_{\alpha}^{2}+Q^{2}}\right)^{2}
$$

Choosing $a_{\alpha}$ to be a negative constant makes this force purely attractive. The parameters

of this force, which we name MT3-I in the following, are given in Table II. They are chosen to give a small attractive contribution to the three-body binding energy, such that we end 
up in the neighborhood of the triton binding energy. For the force parameters in Table II we obtain $E=8.8732 \mathrm{MeV}$.

In order to solve the eigenvalue equation, Eq. (2.10), for the Faddeev component $\psi(p, q, x)$, we use Gaussian grid points in $p, q$, and $x$. The momentum and angle grids in the integrations of the 3BF in Eqs. (2.23) and (2.27) have the same sizes as the ones for $p, q$, and $x$. This is very reasonable, since the integrations over the meson exchange contributions of the $3 \mathrm{BF}$, Eq. (2.23), require grids similar in range to the one used to calculate the two-body t-matrix. The p-grid is defined between 0 and $p_{\max }=60 \mathrm{fm}^{-1}$, whereas for the q-grid a maximum value $q_{\max }=40 \mathrm{fm}^{-1}$ is sufficient. For the angle $(x)$ integration, the preferred number is 42 grid points. Further details concerning the grid choices are given in Ref. [12]. Typical grid sizes are $97 \times 97 \times 42$ to obtain an accuracy in the binding energy of 5 significant figures. The convergence of the three-body binding energy $E$ as function of the number of grid points is shown in Table III, where we see a convergence of the energy eigenvalue and the expectation values within 5 digits.

The three-body wave function is calculated from the Faddeev component using Eq. (2.29). Since this wave function enters the eigenvalue equation, Eq. (2.10), we also need to worry about the quality of the calculation of $\Psi(p, q, x)$. One check of the overall quality of the wave function is a comparison of the expectation value of the total Hamiltonian $\langle H\rangle$ with the calculated value $E$ from the solution of the Faddeev equation. Explicitly, we evaluate

$$
\langle H\rangle \equiv\langle\Psi|H| \Psi\rangle=\left\langle\Psi\left|H_{0}\right| \Psi\right\rangle+\left\langle\Psi\left|V_{I I}\right| \Psi\right\rangle+\left\langle\Psi\left|V_{123}\right| \Psi\right\rangle
$$

where $V_{I I}$ represents the $2 \mathrm{BF} \sum_{i=1}^{3} V^{i}$ and $V_{123}$ the three-body defined in Eq. (2.2). The expectation value of the kinetic energy $\left\langle H_{0}\right\rangle$ and the two-body potential energy $\left\langle V_{I I}\right\rangle$ are given as [12]

$$
\begin{aligned}
\left\langle H_{0}\right\rangle & \equiv\left\langle\Psi\left|H_{0}\right| \Psi\right\rangle=3\left\langle\psi\left|H_{0}\right| \Psi\right\rangle \\
& =3 \cdot 8 \pi^{2} \int_{0}^{\infty} p^{2} d p \int_{0}^{\infty} q^{2} d q\left(\frac{p^{2}}{m}+\frac{3 q^{2}}{4 m}\right) \int_{-1}^{+1} d x \psi(p, q, x) \Psi(p, q, x)
\end{aligned}
$$

and

$$
\begin{aligned}
\left\langle V_{I I}\right\rangle & \equiv\left\langle\Psi\left|V_{I I}\right| \Psi\right\rangle=3\left\langle\Psi\left|V^{1}\right| \Psi\right\rangle \\
& =3 \cdot 8 \pi^{2} \int_{0}^{\infty} p^{2} d p \int_{0}^{\infty} q^{2} d q \int_{-1}^{+1} d x \int_{0}^{\infty} p^{2} d p^{\prime} \int_{-1}^{+1} d x^{\prime} \\
& \times \Psi(p, q, x) v_{1}\left(p, p^{\prime}, x, x^{\prime}\right) \Psi\left(p^{\prime}, q, x^{\prime}\right)
\end{aligned}
$$

where

$$
v_{1}\left(p, p^{\prime}, x, x^{\prime}\right)=\int_{0}^{2 \pi} d \phi V^{1}\left(p, p^{\prime}, x x^{\prime}+\sqrt{1-x^{2}} \sqrt{1-x^{2}} \cos \phi\right) .
$$

The expectation value of the three-body potential energy, $\left\langle V_{123}\right\rangle$, is given by

$$
\begin{aligned}
\left\langle V_{123}\right\rangle & \equiv\left\langle\Psi\left|V_{123}\right| \Psi\right\rangle=3\left\langle\Psi\left|V_{4}\right| \Psi\right\rangle \\
& =3 \cdot 8 \pi^{2} \int_{0}^{\infty} p^{2} d p \int_{0}^{\infty} q^{2} d q \int_{-1}^{+1} d x \Psi(p, q, x) V_{4} \Psi(p, q, x) .
\end{aligned}
$$


Here the integrations need the evaluation of the matrix element $\left\langle\mathbf{p q}\left|V_{4}\right| \Psi\right\rangle$ of Eq. (2.14). The expectation values of the kinetic and potential energies are listed in Table III as functions of the size of the $p-q-x$ grid. Table III shows that the expectation values given above converge for 5 significant figures when the grid sizes for $p$ and $q$ exceed 77 points. However, Table III also shows that the expectation value $\langle H\rangle$ does not exactly converge to the calculated value $E$ despite increased grid size. The difference between the two quantities is of the order of $10 \mathrm{keV}$. This behavior is in contrast to calculations based solely on two-body forces. Calculations with $V_{123}=0$ are shown in Table IV as function of the size of the $p-q-x$ grid. Here the convergence of $\langle H\rangle$ to the calculated value $E$ as function of the grid size is much better than in the case where an attractive $3 \mathrm{BF}$ is included.

It is well known that three nucleon forces based on multi-meson exchanges can have attractive as well as repulsive pieces. Thus we also consider a model of this type given as

$$
\begin{aligned}
V_{4} & =\frac{1}{(2 \pi)^{6}} \frac{a_{\alpha}}{m_{\alpha}} g_{\alpha}^{2} \frac{F_{\alpha}\left(Q^{2}\right)}{Q^{2}+m_{\alpha}^{2}} \frac{F_{\alpha}\left(Q^{\prime 2}\right)}{Q^{\prime 2}+m_{\alpha}^{2}} \\
& +\frac{1}{(2 \pi)^{6}} \frac{a_{\alpha \rho}}{\sqrt{m_{\alpha} m_{\rho}}} g_{\alpha} g_{\rho}\left(\frac{F_{\alpha}\left(Q^{2}\right)}{Q^{2}+m_{\alpha}^{2}} \frac{F_{\rho}\left(Q^{\prime 2}\right)}{Q^{\prime 2}+m_{\rho}^{2}}+\frac{F_{\rho}\left(Q^{\prime 2}\right)}{Q^{\prime 2}+m_{\rho}^{2}} \frac{F_{\alpha}\left(Q^{2}\right)}{Q^{2}+m_{\alpha}^{2}}\right) .
\end{aligned}
$$

Here the first term represents an attractive force, characterized by a negative coupling $a_{\alpha}$, whereas the second term represent a repulsive force, i.e. $a_{\alpha \rho}$ is positive. Since the masses of the exchange mesons are different, the form of the second, repulsive term guarantees that $V_{4}$ is symmetric under a permutation of nucleons 2 and 3 . The cutoff functions $F_{\alpha}$ and $F_{\rho}$ have the same functional form as given in Eq. (3.3). The parameters of this 3BF, named MT3-II in the following, are given in Table V. They are chosen so that the correction due to this $3 \mathrm{BF}$ to the three-body binding energy calculated with the $2 \mathrm{BF}$ MT2-II is small. The binding energy $E$ with this MT3-II 3BF gives $E=8.6478 \mathrm{MeV}$.

The expectation values of the kinetic and potential energies are listed in Table VI as functions of the size of the $p-q-x$ grid. Again, the expectation values converge within five significant figures when the grid sizes for $p$ and $q$ exceed 77 points. However, now the difference of the expectation value for the total Hamiltonian $\langle H\rangle$ deviates from the calculated eigenvalue $E$ only by $5 \mathrm{keV}$, a number being similar to calculations carried out in a partial wave decomposition and based on realistic forces [22].

All these numbers are not meant to provide insight into the physics of three interacting nucleons, but serve only as a demonstration that this technique allows a very accurate and easy handling of typical nuclear forces consisting of attractive and repulsive (short range) parts. In addition, they will serve as benchmarks for future studies.

\section{WAVE FUNCTION PROPERTIES}

Despite the fact that there is no spin dependence and questions about effects of realistic forces can not be posed, we want to display some wave function properties, which are often studied in the context of realistic forces. It will turn out that qualitatively they also appear in our simple three boson model. The probability of finding a nucleon with momentum $q$ in the nucleus is given as 


$$
n(q)=2 \pi q^{2} \int_{0}^{\infty} d p p^{2} \int_{-1}^{+1} d x \Psi^{2}(p, q, x)
$$

The total wave function $\Psi(p, q, x)$ is given by Eq. (2.29). In Fig. 2 we show $n(q)$ with and without three-body forces. There is hardly any change, a fact with has been noticed before in the context of realistic nuclear forces [22]. In addition, the shoulder of the distribution around 2-4 $\mathrm{fm}^{-1}$ is qualitatively similar to the case when using realistic forces. The momentum distribution $n(q)$ is shown in Fig. 2 for three different cases, one based on a calculation with two-body forces alone (dotted line), and the other two for which the two different 3BFs are included. First we notice that the two 3BF's, though different in character, lead to essentially the same momentum distribution. Compared to the momentum distribution given by the two-body force alone, the minimum is shifted to a slightly higher momentum.

Another property often investigated is the probability to find two nucleons at a distance $r$. To obtain that quantity we generate the total wave function in configuration space as

$$
\Psi(\mathbf{r}, \mathbf{R})=\int d^{3} p d^{3} q \Psi(\mathbf{p}, \mathbf{q}) \exp (i \mathbf{p} \cdot \mathbf{r}) \exp (i \mathbf{q} \cdot \mathbf{R})
$$

Here $\Psi(\mathbf{p}, \mathbf{q})$ is the total wave function in momentum space as given by Eq. (2.6). The variables $\mathbf{r}$ and $\mathbf{R}$ are conjugate to the Jacobi momenta $\mathbf{p}, \mathbf{q}$ and given as

$$
\begin{aligned}
\mathbf{r} & =\mathbf{x}_{2}-\mathbf{x}_{3}, \\
\mathbf{R} & =\mathbf{x}_{1}-\frac{1}{2}\left(\mathbf{x}_{2}+\mathbf{x}_{3}\right) .
\end{aligned}
$$

where $\mathbf{x}_{1}, \mathbf{x}_{2}$ and $\mathbf{x}_{3}$ are the coordinates of three nucleons in configuration space [16].

For the explicit calculation of the double Fourier transformation we first consider the q-integration

$$
\int d^{3} q \exp (i \mathbf{q} \cdot \mathbf{R}) \Psi(\mathbf{p}, \mathbf{q})
$$

We choose the vector $\mathbf{p}$ parallel to the z-axis and define the angles $\hat{\mathbf{q}} \cdot \hat{\mathbf{z}}=x_{q}$ and $\hat{\mathbf{R}} \cdot \hat{\mathbf{z}}=x_{R}$. Since the integration is carried out over all space, we can set $\phi_{R}=0$, and obtain

$$
\begin{aligned}
& \int d^{3} q \exp (i \mathbf{q} \cdot \mathbf{R}) \Psi(\mathbf{p}, \mathbf{q}) \\
& =\int_{0}^{\infty} q^{2} d q \int_{-1}^{+1} d x_{q} \int_{0}^{2 \pi} d \phi_{q} \exp (i q R \hat{\mathbf{R}} \cdot \hat{\mathbf{q}}) \Psi\left(p, q, x_{q}\right),
\end{aligned}
$$

where

$$
\hat{\mathbf{R}} \cdot \hat{\mathbf{q}}=x_{q} x_{R}+\sqrt{1-x_{q}^{2}} \sqrt{1-x_{R}^{2}} \cos \phi_{q}
$$

Thus, the integration over $\phi_{q}$ can be carried out separately

$$
\begin{aligned}
& \int_{0}^{2 \pi} d \phi_{q} \exp \left(i q R \sqrt{1-x_{q}^{2}} \sqrt{1-x_{R}^{2}} \cos \phi_{q}\right) \\
& =2 \pi J_{0}\left(q R \sqrt{1-x_{q}^{2}} \sqrt{1-x_{R}^{2}} \cos \phi_{q}\right) .
\end{aligned}
$$


Summarizing the above leads to the intermediate result

$$
\begin{aligned}
& \int d^{3} q \exp (i \mathbf{q} \cdot \mathbf{R}) \mathbf{\Psi}(\mathbf{p}, \mathbf{q}) \\
& =2 \pi \int_{0}^{\infty} q^{2} d q \int_{-1}^{+1} d x_{q} J_{0}\left(q R \sqrt{1-x_{q}^{2}} \sqrt{1-x_{R}^{2}} \cos \phi_{q}\right) \exp \left(i q R x_{q} x_{R}\right) \Psi\left(p, q, x_{q}\right) \\
& \equiv 2 \pi \Psi_{p}\left(p, R, x_{R}\right) .
\end{aligned}
$$

Next, we consider the integration over $\mathbf{p}$, where it is convenient to choose the vector $\mathbf{r}$ parallel to the z-axis. Thus, the following angle, $\hat{\mathbf{R}} \cdot \hat{\mathbf{z}} \equiv \hat{\mathbf{R}} \cdot \hat{\mathbf{r}} \equiv x_{R}$, needs to be considered, and the integration over $x_{p}$ and $\phi_{p}$ can be carried out separately as

$$
\int_{-1}^{+1} d x_{p} \int_{0}^{2 \pi} d \phi_{p} \exp \left(i p r x_{p}\right)=4 \pi \frac{\sin (p r)}{p r}
$$

Finally, the Fourier transform of $\Psi(p, q, \hat{\mathbf{p}} \cdot \hat{\mathbf{q}})$ can be calculated as

$$
\Psi\left(r, R, x_{R}\right)=\frac{8 \pi^{2}}{r} \int_{0}^{\infty} d p\left[\sin (p r) p \Psi_{p}\left(p, R, x_{R}\right)\right]
$$

where

$$
\Psi_{p}\left(p, R, x_{R}\right)=\int_{0}^{\infty} q^{2} d q \int_{-1}^{+1} d x_{q} J_{0}\left(q R \sqrt{1-x_{q}^{2}} \sqrt{1-x_{R}^{2}}\right) \cos \left(q R x_{q} x_{R}\right) \Psi\left(p, q, x_{q}\right) .
$$

With this the two-body correlation function $c(r)$ is defined as

$$
c(r)=2 \pi r^{2} \int_{0}^{\infty} d R R^{2} \int_{-1}^{+1} d x_{R} \Psi^{2}\left(r, R, x_{R}\right)
$$

The correlation function $c(r)$ describes the probability to find two nucleons within a relative distance $r$. In Fig. 3 the correlation functions are displayed based on a calculation with twobody forces (dotted line) and based on calculations with the two different 3BF's. Though our model is very simple, the functions $c(r)$ are similar to the ones obtained with realistic forces [22]. We see that the maximum of $c(r)$ is shifted slightly to a smaller value of $r$ once a $3 \mathrm{BF}$ is included, which is consistent with the minimum of $n(q)$ being shifted to a slightly higher momentum. The position of the maximum of $c(r)$ does not depend on the type of 3BF, however the actual shape of the function does.

Since our three-body system consists of three identical nucleons acted on by scalar forces, the three nucleons form a ground state where the most probable positions of the nucleons have the shape of an equilateral triangle. The expectation values of the Jacobi coordinates $r$ and $R$ can be calculated as

$$
\begin{aligned}
\langle r\rangle & =\int_{0}^{\infty} R^{2} d R \int_{0}^{\infty} r^{2} d r \int_{-1}^{+1} d x_{R} r \Psi^{2}\left(r, R, x_{R}\right), \\
\langle R\rangle & =\int_{0}^{\infty} R^{2} d R \int_{0}^{\infty} r^{2} d r \int_{-1}^{+1} d x_{R} R \Psi^{2}\left(r, R, x_{R}\right) .
\end{aligned}
$$

Here the values $\langle r\rangle$ and $\langle R\rangle$ are the length and height of the equilateral triangle. The geometrical relation between the length and height of an equilateral triangle is given by 


$$
\langle r\rangle:\langle R\rangle=2: \sqrt{3}
$$

We also define a deviation $\delta$ by

$$
\delta=\frac{\langle r\rangle /\langle R\rangle-2 / \sqrt{3}}{2 / \sqrt{3}} \times 100 .
$$

to account for deviations from an ideal geometric triangle. In Table VII we show the corresponding values for $\langle r\rangle,\langle R\rangle$, and $\delta$ for the MT2-II 2BF alone and the cases where the two different $3 \mathrm{BF}$ discussed above are added. In all cases listed, the deviation from an ideal equilateral triangle is $3 \%$ or less, also indicating that our calculations are very accurate.

We would like to add a little excursion into a playground with forces. Assume there are only three-body forces. Can one generate a three-body wave function which has about the same binding energy, single nucleon momentum distribution, and two-body correlation function as given by two-body forces alone? This is indeed possible. For that aim we have chosen purely attractive two- and three-body forces, the parameters of which are given in Table VIII. The binding energies and expectation values of kinetic and potential energies for the two cases are displayed in Table IX. It turns out that also $n(q)$ and $c(r)$ are close to each other as shown in Figs. 3 and 4 . We do not know of a physical realization of such a scenario with pure three-body forces, but maybe the reader may find that little excursion equally entertaining as we do.

\section{SUMMARY AND OUTLOOK}

We derived and calculated the Faddeev equation for three identical bosons interacting by two- and three-body forces. The equation is formulated in momentum space directly in terms of momentum vectors, i.e. without angular momentum decomposition. It is demonstrated that this equation can be solved by integrating over magnitudes of momenta and various angles. In doing so we encounter interpolations which are carried out by cubic Hermitian splines [18]. The Faddeev equation is solved by iteration using a Lanczo's type method [4,24]. An accuracy of 5 digits in the energy eigenvalue can easily be achieved. In comparison to an angular momentum decomposition which is commonly used [16], this direct approach has great advantages. It avoids the very involved angular momentum algebra occurring for the permutations and especially for the three-body forces $[17,18]$.

All two- and three-body forces we employ are of meson exchange type, either purely attractive or attractive and repulsive. The mesons responsible for the attraction have masses around $300 \mathrm{MeV}$, the ones for the repulsion around $600 \mathrm{MeV}$. These forces, serving as a reference, are chosen with a view towards nuclear physics. Thus the reference two-body forces lead to a three-body binding energy somewhat smaller than $8.48 \mathrm{MeV}$ (the experimental ${ }^{3} \mathrm{H}$ binding energy), and the reference three-body forces add about $1 \mathrm{MeV}$ additional binding energy.

For these type of forces we evaluated the single nucleon momentum distributions and the two-body correlation functions. These quantities turned out to be qualitatively very similar to what is achieved with realistic spin dependent forces $[22,23]$.

We also evaluated the expectation values $\langle r\rangle$ for a pair distance and $\langle R\rangle$ for the distance of a third particle to the c.m. of the corresponding pair. In case of an equilateral triangle 
the ratio of these two quantities is $2 / \sqrt{3}$. Since we have three identical bosons the position of the three particles in the ground state should be with highest probability at the corners of an equilateral triangle. Indeed the corresponding ratio for the expectation values $\langle r\rangle$ and $\langle R\rangle$ turned out to be $2 / \sqrt{3}$ with an numerical error of about $1-2 \%$.

Including spin (and isospin) degrees of freedom is an additional task for the future, which will increase the space of states and will lead to coupled equations, but only a strictly finite number of equations. The form in which they will appear will depend on the way the spin degrees of freedom will be incorporated. One possibility will be the extension of the helicity formalism chosen for the NN system in a three-dimensional notation [15] to three nucleons. The other possible extension of the here presented formulation is the incorporation of relativity in the instant form of dynamics [25]. The momentum space formulation seems ideal for that. First steps have been already undertaken [26,27]. Since relativity will be of importance at higher energies our treatment with momentum vectors and avoiding angular momentum decomposition will be awarding in that respect.

\section{ACKNOWLEDGMENTS}

This work was performed in part under the auspices of the U. S. Department of Energy under contract No. DE-FG02-93ER40756 with Ohio University. The computational support of the Ohio Supercomputer Center (OSC) for the use of their facilities under Grant No. PHS206, the Neumann Institute for Computing (NIC) under project JIKP01 and the

National Energy Research Supercomputer Center (NERSC) is acknowledged. The authors want to thank A. Nogga for helpful and stimulating discussions. 


\section{REFERENCES}

[1] R.A. Malfliet, A.J. Tjon, Nucl. Phys. A, 127, 161 (1969).

[2] T.A. Osborn, 'Faddeevs Equations for local potentials, SLAC Report No. 17 (1967).

[3] A. Picklesimer, R.A. Rice, and R. Brandenburg, Phys. Rev C45, 2045 (1992), ibid, 547 (1992), Phys. Rev. C44, 1359 (1991).

[4] A. Stadler, W. Glöckle, P.U. Sauer, Phys. Rev. C44, 2319 (1991);

[5] A. Stadler and P.U. Sauer, Phys. Rev. C46, 64 (1992).

[6] A. Nogga, D. Hüber, H. Kamada, W. Glöckle, Phys. Lett. B 409, 19 (1997).

[7] A. Laverne, C. Gignoux, Nucl. Phys. A203, 597 (1973).

[8] J.L. Friar, B.F. Gibson, and G.L. Payne, Z. Phys. A 301, 309 (1981), C.R. Chen, G.L. Payne, J.L. Friar, B.F. Gibson, Phys. Rev. C31, 2266 (1985).

[9] N.W. Schellingerhout, L.P. Kok, and G.D. Bosveld, Phys. Rev. A40, 5568 (1989); N.W. Schellingerhout, J.J. Schut, and L.P. Kok, Phys. Rev. C46, 1192 (1992).

[10] Y. Wu, S. Ishikawa, T. Sasakawa, Few-Body Systems 15, 145 (1993).

[11] J. Carlson, Phys. Rev. C36, 2026 (1987), Phys. Rev. C38, 1879 (1988); J.G. Zabolitzki, K.E. Schmidt, and M.H. Kalos, Phys. Rev. C25, 1111 (1982); J. Carlson and R. Schiavilla, Rev. Mod. Phys. 70, 743 (1998).

[12] Ch. Elster, W. Schadow, A. Nogga, and W. Glöckle, Few-Body System, 27, 83 (1998).

[13] W. Schadow, Ch. Elster, and W. Glöckle, Few-Body Systems 28, 15 (2000).

[14] A.A. Kvitsinsky and C.-Y. Hu, Few-Body Systems 12, 7 (1992).

[15] I. Fachruddin, Ch. Elster, W. Glöckle, Phys. Rev. C62, 044002-1 (2000).

[16] W. Glöckle, The Quantum Mechanical Few-Body Problem, Springer-Verlag (1983).

[17] S.A. Coon and W. Glöckle, Phys. Rev. C23, 1790 (1981).

[18] D. Hüber, H. Witala, A. Nogga, W. Glöckle and H. Kamada, Few-Body System, 22, 107 (1997).

[19] J. Fujita, H. Miyazawa, Prog. Theor. Phys. 17, 360 (1957).

[20] Ch. Elster, E.E. Evans, H. Kamada and W. Glöckle, Few-Body System, 21, 25 (1996).

[21] Ch. Elster, J.H. Thomas, W. Glöckle, Few-Body Systems, 24, 55 (1998).

[22] A. Nogga, PhD Thesis, Ruhr-University Bochum, 2001.

[23] A. Nogga, D. Hüber, H. Kamada, W. Glöckle, Phys. Lett. B409, 19 (1997).

[24] A. Saake, Diploma Thesis, Ruhr-University Bochum, 1992 (unpublished).

[25] W. Glöckle, T.-S.H. Lee, F. Coester, Phys. Rev. C33, 709 (1986).

[26] H. Kamada, Few-Body Systems, Suppl. 12, 433 (2000).

[27] H. Kamada, W. Glöckle, J. Golak, Ch. Elster, Phys. Rev. C66, 044010 (2002). 


\section{TABLES}

TABLE I. The parameters of the MT2-II two-body force.

\begin{tabular}{cccccc}
\hline \hline$g_{A}^{2} / 4 \pi$ & $m_{A}[\mathrm{MeV}]$ & $\Lambda_{A}[\mathrm{MeV}]$ & $g_{R}^{2} / 4 \pi$ & $m_{R}[\mathrm{MeV}]$ & $\Lambda_{R}[\mathrm{MeV}]$ \\
\hline 3.5775 & 330.2104 & 1500.0 & 9.4086 & 612.4801 & 1500.0 \\
\hline \hline
\end{tabular}

TABLE II. The parameters of the MT3-I attractive 3BF.

\begin{tabular}{lccc}
\hline \hline$g_{\alpha}^{2} / 4 \pi$ & $m_{\alpha}[\mathrm{MeV}]$ & $\Lambda_{\alpha}[\mathrm{MeV}]$ & $a_{\alpha}$ \\
\hline 5.0 & 305.8593 & 1000.0 & -1.73 \\
\hline \hline
\end{tabular}

TABLE III. The calculated eigenvalue $E$ from the the solution of the Faddeev equation and the expectation values of the kinetic energy $\left\langle H_{0}\right\rangle$, the two-body potential $\left\langle V_{I I}\right\rangle$, the three-body potential energy $\left\langle V_{123}\right\rangle$ and the total Hamiltonian $\langle H\rangle$ as functions of the number of grid points NP, NQ and NX for the $p-q-x$ grid. The calculations are based on the MT2-II 2BF and the MT3-I 3BF.

\begin{tabular}{lccccccr}
\hline \hline $\mathrm{NP}$ & $\mathrm{NQ}$ & $\mathrm{NX}$ & $\left\langle H_{0}\right\rangle(\mathrm{MeV})$ & $\left\langle V_{I I}\right\rangle(\mathrm{MeV})$ & $\left\langle V_{123}\right\rangle(\mathrm{MeV})$ & $\langle H\rangle(\mathrm{MeV})$ & $E(\mathrm{MeV})$ \\
\hline 45 & 45 & 42 & 31.8838 & -39.4069 & -1.3392 & -8.8623 & -8.8715 \\
\hline 61 & 45 & 42 & 31.8846 & -39.4073 & -1.3396 & -8.8623 & -8.8711 \\
\hline 77 & 45 & 42 & 31.8848 & -39.4074 & -1.3397 & -8.8623 & -8.8709 \\
\hline 77 & 61 & 42 & 31.8900 & -39.4133 & -1.3406 & -8.8639 & -8.8726 \\
\hline 77 & 77 & 42 & 31.8915 & -39.4149 & -1.3409 & -8.8643 & -8.8731 \\
\hline 87 & 87 & 42 & 31.8919 & -39.4152 & -1.3410 & -8.8644 & -8.8732 \\
\hline 97 & 97 & 42 & 31.8920 & -39.4154 & -1.3410 & -8.8644 & -8.8732 \\
\hline \hline
\end{tabular}

TABLE IV. The calculated eigenvalue $E$ of the Faddeev equation and the expectation values of the kinetic energy $\left\langle H_{0}\right\rangle$, the two-body potential $\left\langle V_{I I}\right\rangle$, and the total Hamiltonian $\langle H\rangle$ as functions of the number of grid points NP, NQ and NX for the $p-q-x$ grid. The calculations are based on the MT2-II 2BF alone.

\begin{tabular}{lcccccc}
\hline \hline $\mathrm{NP}$ & $\mathrm{NQ}$ & $\mathrm{NX}$ & $\left\langle H_{0}\right\rangle(\mathrm{MeV})$ & $\left\langle V_{I I}\right\rangle(\mathrm{MeV})$ & $\langle H\rangle(\mathrm{MeV})$ & $E(\mathrm{MeV})$ \\
\hline 77 & 77 & 42 & 28.6408 & -36.3390 & -7.6983 & -7.6984 \\
\hline 87 & 87 & 42 & 28.6408 & -36.3391 & -7.6983 & -7.6984 \\
\hline 97 & 97 & 42 & 28.6408 & -36.3392 & -7.6983 & -7.6984 \\
\hline \hline
\end{tabular}

TABLE V. The parameters of the MT3-II 3BF.

\begin{tabular}{cccc}
\hline \hline$g_{\alpha}^{2} / 4 \pi$ & $m_{\alpha}[\mathrm{MeV}]$ & $\Lambda_{\alpha}[\mathrm{MeV}]$ & $a_{\alpha}$ \\
\hline 5.0 & 305.8593 & 1000.0 & -2.69 \\
\hline$g_{\rho}^{2} / 4 \pi$ & $m_{\rho}[\mathrm{MeV}]$ & $\Lambda_{\rho}[\mathrm{MeV}]$ & $a_{\alpha \rho}$ \\
\hline 9.0 & 650.0000 & 1900.0 & 2.40 \\
\hline \hline
\end{tabular}


TABLE VI. The calculated eigenvalue $E$ from the the solution of the Faddeev equation and the expectation values of the kinetic energy $\left\langle H_{0}\right\rangle$, the two-body potential $\left\langle V_{I I}\right\rangle$, the three-body potential energy $\left\langle V_{123}\right\rangle$ and the total Hamiltonian $\langle H\rangle$ as functions of the number of grid points NP, NQ and NX for the $p-q-x$ grid. The calculations are based on the MT2-II 2BF and the MT3-II 3BF.

\begin{tabular}{cccccccc}
\hline \hline $\mathrm{NP}$ & $\mathrm{NQ}$ & $\mathrm{NX}$ & $\left\langle H_{0}\right\rangle(\mathrm{MeV})$ & $\left\langle V_{I I}\right\rangle(\mathrm{MeV})$ & $\left\langle V_{123}\right\rangle(\mathrm{MeV})$ & $\langle H\rangle(\mathrm{MeV})$ & $E(\mathrm{MeV})$ \\
\hline 45 & 45 & 42 & 31.1745 & -38.7859 & -1.0404 & -8.6518 & -8.6454 \\
\hline 61 & 45 & 42 & 31.1767 & -38.7878 & -1.0409 & -8.6520 & -8.6456 \\
\hline 77 & 45 & 42 & 31.1773 & -38.7881 & -1.0413 & -8.6521 & -8.6466 \\
\hline 77 & 61 & 42 & 31.1825 & -38.7880 & -1.0478 & -8.6533 & -8.6477 \\
\hline 77 & 77 & 42 & 31.1837 & -38.7886 & -1.0481 & -8.6530 & -8.6480 \\
\hline 87 & 87 & 42 & 31.1842 & -38.7887 & -1.0481 & -8.6526 & -8.6478 \\
\hline 97 & 97 & 42 & 31.1847 & -38.7892 & -1.0481 & -8.6526 & -8.6478 \\
\hline \hline
\end{tabular}

TABLE VII. The binding energy $E$, the expectation values $\langle r\rangle$ and $\langle R\rangle$ calculated with the MT2-II 2BF alone and with the addition of the two different 3BF's described in the text. The deviation $\delta$ characterizes the deviation from the shape of an equilateral triangle and is defined in Eq. (4.15).

\begin{tabular}{cccc}
\hline \hline & MT2-II & MT2-II + MT3-I & MT2-II + MT3-II \\
\hline$E(\mathrm{MeV})$ & -7.6980 & -8.873 & -8.6478 \\
$\langle r\rangle(\mathrm{fm})$ & 2.521 & 2.382 & 2.9401 \\
$\langle R\rangle(\mathrm{fm})$ & 2.221 & 2.096 & 2.5945 \\
$\delta(\%)$ & 1.7 & 1.6 & 1.9 \\
\hline \hline
\end{tabular}

TABLE VIII. The parameters of the purely attractive potentials, the $2 \mathrm{BF}$ MT2-I and the $3 \mathrm{BF}$ MMT3-I leading to the same three-body binding energy.

\begin{tabular}{lcccc}
\hline \hline MT2-I & $g_{A}^{2} / 4 \pi$ & $m_{A}[\mathrm{MeV}]$ & $\Lambda_{A}[\mathrm{MeV}]$ & \\
\hline & -0.7210 & 330.2104 & 1500 & $a_{\alpha}$ \\
\hline \hline MMT3-I & $g_{\alpha}^{2} / 4 \pi$ & $m_{\alpha}[\mathrm{MeV}]$ & $\Lambda_{\alpha}[\mathrm{MeV}]$ & -1.90015 \\
\hline
\end{tabular}

TABLE IX. The calculated eigenvalue $E$ from the the solution of the Faddeev equation, the expectation values of the kinetic energy $\left\langle H_{0}\right\rangle$, and the potential energy $\langle V\rangle$ for the two-body force MT2-I and the three-body force MMT3-I. Both forces give similar binding energies. The expectation values of $\langle R\rangle$ and $\langle r\rangle$ are also given for both cases.

\begin{tabular}{cccccc}
\hline \hline Model & $\left\langle H_{0}\right\rangle(\mathrm{MeV})$ & $\langle V\rangle(\mathrm{MeV})$ & $\langle R\rangle(\mathrm{fm})$ & $\langle r\rangle(\mathrm{fm})$ & $\mathrm{E}(\mathrm{MeV})$ \\
\hline MT2-I & 66.967 & -74.547 & 1.592 & 1.783 & -7.5803 \\
\hline MMT3-I & 67.306 & -74.895 & 1.521 & 1.698 & -7.5504 \\
\hline \hline
\end{tabular}




\section{FIGURES}

FIG. 1. Diagrammatic representation of the three-body force $V_{4}^{(1)}$. Here particle (1) is singled out by the meson-nucleon amplitude described by the blob. The three-body force is then given according to Eq. (2.2).

FIG. 2. The momentum distributions $n(q)$ calculated with the MT2-II two-body potential (dotted line). The solid line represents the calculation of $n(q)$ with the MT2-II two-body potential and the MT3-I three-body potential, the dashed line the corresponding calculation with the MT3-II three body potential.

FIG. 3. The two-body correlation function $c(r)$ calculated with the MT2-II two-body potential (dotted line). The solid line represents the calculation of $c(r)$ with the MT2-II two-body potential and the MT3-I three-body potential, the dashed line the corresponding calculation with the MT3-II three body potential.

FIG. 4. The momentum distributions $n(q)$ calculated with the attractive 2BF MT2-I (solid line). The dashed curve represents the calculation with the purely attractive 3BF, where the parameters are chosen such that the binding energy and the momentum distribution are similar to the one given by the MT2-I potential.

FIG. 5. The two-body correlation function $c(r)$ calculated with the attractive 2BF MT2-I (solid line). The dashed curve represents the calculation with the purely attractive 3BF as used in Fig. 4 
Fig.1

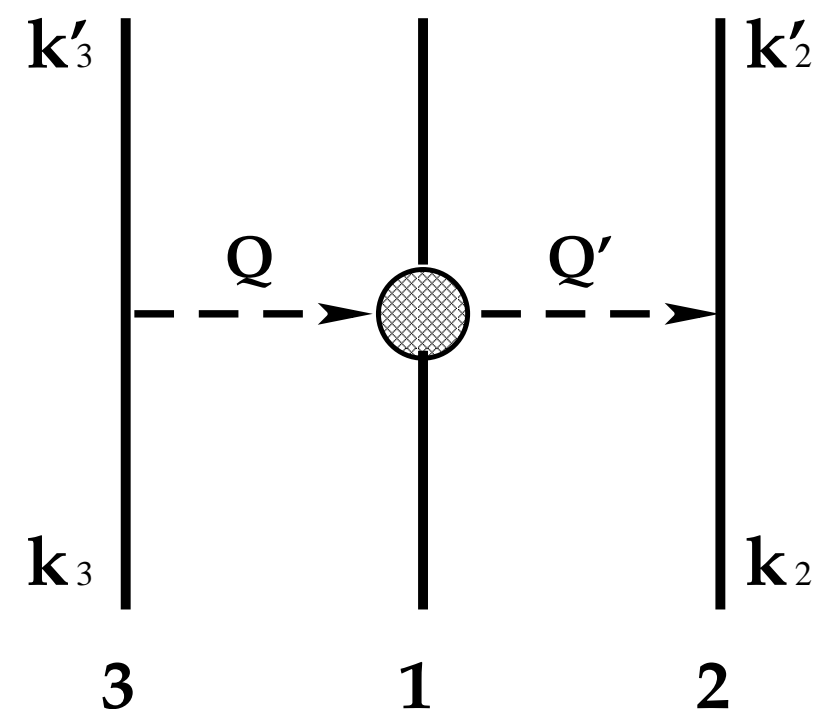

Fig.2

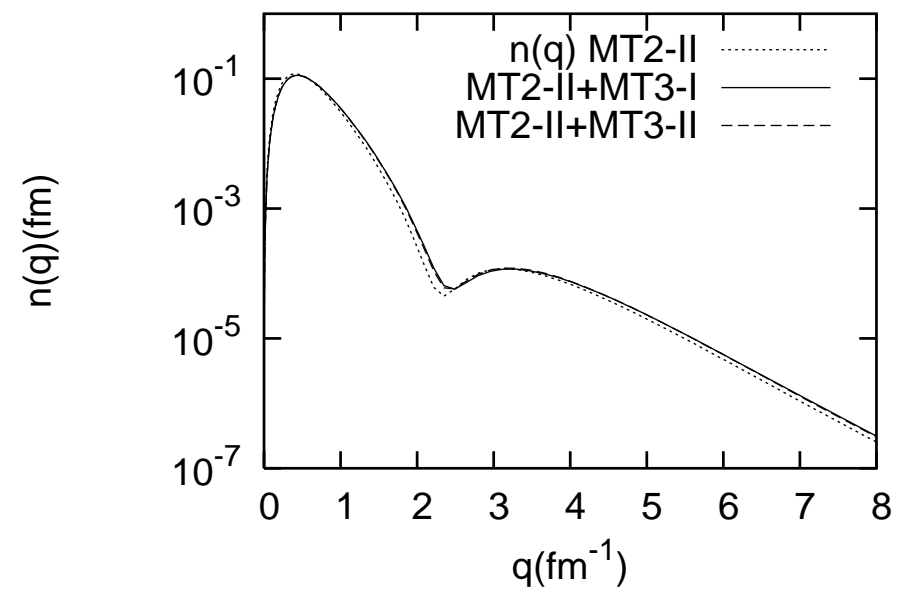


Fig.3

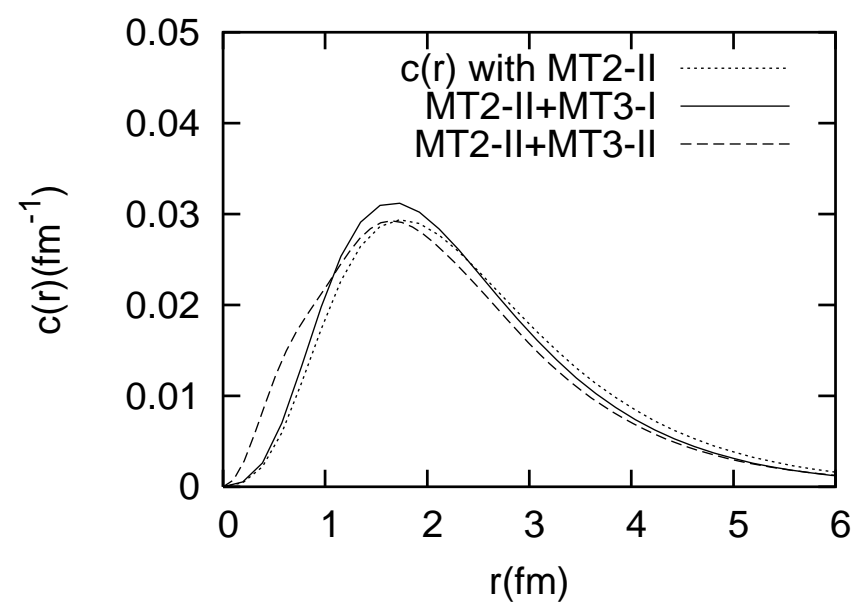

Fig.4

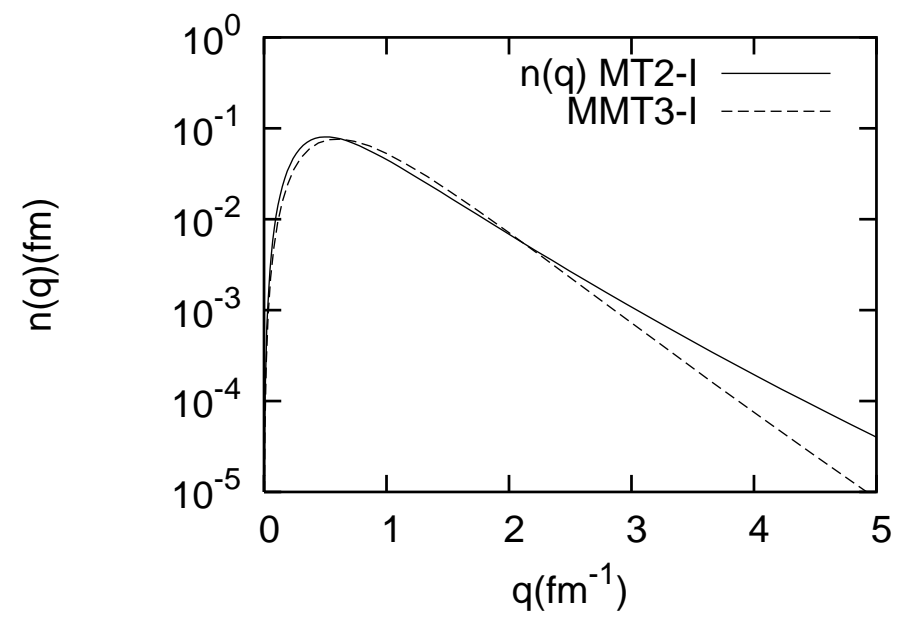

Fig.5

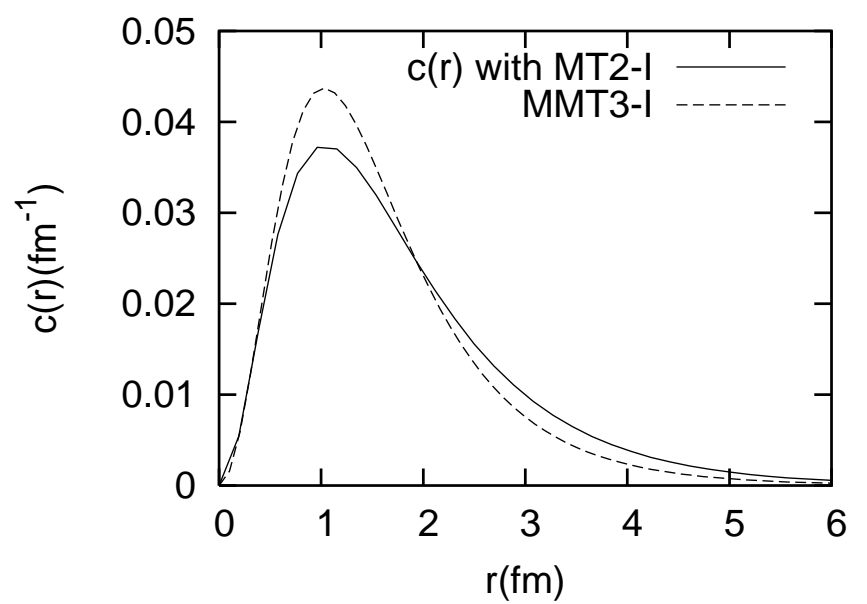

